\title{
Herramientas en la educación del niño asmático
}

\author{
I. Moneo Hernández $z^{\mathrm{a}}$, MP. Oliván Otal ${ }^{\mathrm{b}}$, MD. Forés Catalác, E. Lambán Casamayor ${ }^{c}$ \\ aPediatra. CS Las Fuentes Norte. Zaragoza. España. \\ ${ }^{b}$ Pediatra. CS Canal Imperial. EAP San José Sur. Zaragoza. España. \\ 'Enfermera. CS Las Fuentes Norte. Zaragoza. España.
}

Rev Pediatr Aten Primaria. 2009; I I (Supl I7):s4 I5-s422

Isabel Moneo Hernández, mimoneo@salud.aragon.es

\begin{abstract}
Resumen
La educación es reconocida como un pilar básico en el tratamiento del asma infantil. A todos los niños con asma y sus familias se les debe ofertar en las consultas de Atención Primaria un programa educativo, progresivo, continuo, gradual e individualizado, que tendrá como fin conseguir el control de la enfermedad de la manera más autónoma posible por parte del niño y/o la familia. Ello implica no solo la transmisión de conocimientos, sino la adquisición de habilidades y modificaciones en el estilo de vida de las familias.

En el presente taller repasaremos las herramientas de que dispone el pediatra y la enfermera para realizar la educación del niño asmático, las dificultades más habituales que se presentan en la práctica y los recursos que tenemos para solventarlas, todo ello mediante la presentación de casos clínicos concretos que los alumnos discutirán de forma grupal.

Palabras clave: Asma, Infancia, Educación, Atención Primaria.

\section{Abstract}

Education is recognized as fundamental in the treatment of the asthma in childhood.

Primary care must provide a progressive, continual and individualized educational program to all children with asthma and their families which has as its aim to obtain to the control of the disease in the most independent way possible by the child and/or the family. It implies not only, the transmission of knowledge but the acquiring of abilities and changes in family lifestyles.

In the present workshop we will review the tools which are available to the paediatrician and the nurse to educate the asthmatic child, the most common difficulties that appear in the practice, and the resources that we have to resolve them, all of this by the presentation of specific clinical cases that the students will then discuss in groups.
\end{abstract}

Key words: Asthma, Childhood, Education, Primary Care.

Las autoras declaran no presentar posibles conflictos de intereses en relación con la preparación y publicación de este artículo. 


\section{Introducción}

El asma es, tras la obesidad, la segunda enfermedad crónica de los niños españoles, y claramente es la patología crónica que consume un mayor número de recursos y tiempo en la Atención Primaria (AP) de la infancia.

Para mejorar su control se han desarrollado en nuestro país diversos planes de atención en los últimos años; algunos centrados en el primer nivel asistencial ${ }^{1-3}$, otros abarcando también la Atención Especializada y hospitalaria ${ }^{4-6}$ y ha sido la materia para la elaboración de guías de práctica clínica con metodología basada en la evidencia ${ }^{7.8}$ en varias comunidades, en otras como la Navarra han incorporado las guías nacionales ${ }^{9}$ como documento de base para el control de la enfermedad. Es, en definitiva, un tema que nos preocupa y ocupa a todos los pediatras de AP.

\section{¿Qué papel juega la educación en el control del asma?}

En las últimas guías y protocolos nacionales ${ }^{9,10}$ y también en las internacionales ${ }^{11-13}$ se plantea como imprescindible el incluir al niño diagnosticado de asma en un plan educativo. Las últimas recomendaciones de la GINA 2009 son taxativas: "Todos los pacientes asmáticos, si han requerido un ingreso o han realizado una visita a urgencias por una crisis, deberían ser incluidos en un programa de manejo de asma antes de 30 días".

El objetivo que perseguimos con este plan educativo es que el niño y la familia deben aceptar y conocer la enfermedad, y además deben saber cómo actuar en cada momento, es decir, conseguir el autocontrol que les permita una vida lo más parecida posible a la vida en ausencia de enfermedad.

Este plan se debe desarrollar como actividad programada y se ha demostrado con estudios que arrojan el máximo nivel de evidencia que los resultados son más relevantes cuanto mayor sea la gravedad de la enfermedad y si iniciamos el plan educativo inmediatamente tras el diagnóstico. La educación ha demostrado su eficacia con menor nivel de evidencia $1 \mathrm{~B}^{12}$ para niños menores de 5 años, el grupo más heterogéneo, en el que existe una considerable incertidumbre diagnóstica, terapéutica y pronóstica.

\section{¿Quién debe responsabilizarse} de la educación?

El abordaje educativo del niño y adolescente con asma compete a todos los profesionales sanitarios: pediatras, neumólogos, alergólogos, enfermeras/os, fisioterapeutas o farmacéuticos ${ }^{14}$. Se puede ejercer tanto en el ámbito de la atención hospitalaria, como en la AP. 
Los profesionales de Pediatría y Enfermería de AP por su posición de cercanía, accesibilidad y confianza, así como por la posibilidad de un seguimiento cercano y continuado, son los mejor situados para implicarse en la educación del niño y de la familia del asmático.

La Enfermería de AP ha adquirido un papel principal como proveedora de planes de cuidados y su intervención en otras enfermedades crónicas como la diabetes, ha demostrado una elevada eficacia; por ello, tiene un papel fundamental en la educación de los niños asmáticos. Para desarrollarla adecuadamente debe estar motivada y formada, y ha de disponer de tiempo y de recursos necesarios para ello ${ }^{15}$.

\section{¿Qué contenidos debemos incluir en el programa educativo?}

El plan educativo debe ser gradual y progresivo, individualizado y adaptado a la capacidad de cada niño y su familia (tabla I).

Existen una serie de conocimientos y habilidades mínimos que todos los pacientes deben conocer:

- Concepto de asma: es una enfermedad crónica inflamatoria de los bronquios, con una base genética, que puede sufrir empeoramientos en forma de crisis.

- Cuáles son los síntomas que anuncian una crisis y cómo actuar frente a ella.

- Factores desencadenantes de crisis, saber cuáles son los suyos, aprender a evitarlos y a reconocerlos.

- Cómo se usan los distintos sistemas de inhalación.

- La diferencia entre la medicación de rescate para el tratamiento de la crisis y la medicación de control.

\begin{tabular}{|c|c|c|}
\hline \multicolumn{3}{|c|}{ Tabla I. Manejo en fases escalonadas } \\
\hline Autocontrol & Para algunos casos & $\begin{array}{l}\text { - Manejo del PEF. Registro de síntomas } \\
\text { - } \quad \text { Autocontrol. Toma de decisiones autónomas. } \\
\text { - Plan de cuidados, escrito. }\end{array}$ \\
\hline Compartida & Para la mayoría & $\begin{array}{l}\text { - } \quad \text { Conocimientos ampliados. Estilo de vida. } \\
\text { - Identificación de desencadenantes específicos. } \\
\text { - Itinerarios terapéuticos. } \\
\text { - Tratamiento precoz de la crisis. Plan de cuidados, escrito. }\end{array}$ \\
\hline Básica & Para todos & $\begin{array}{l}\text { - Conocimientos básicos. Síntomas. } \\
\text { - Tratamiento y técnica de inhalación. } \\
\text { - Medidas generales de evitación. } \\
\text { - Crisis. Plan de cuidados, escrito. }\end{array}$ \\
\hline
\end{tabular}


- Cuáles son los síntomas de gravedad y cuándo deben pedir ayuda médica.

Debemos entregar a todos los pacientes un plan por escrito. En este plan debe de estar explicado de forma comprensible qué medicación debe tomar de forma diaria, cuándo debe usar medicación de rescate, en qué cantidad y con qué sistema y cuándo deben solicitar atención médica, este proceso sirve para que el niño y los cuidadores relacionen la aparición de los síntomas, los factores desencadenantes y el uso de la medicación.

Los planes de acción por escrito han demostrado ser eficaces para la mejora de la función pulmonar, para disminuir el número de ausencias de la escuela y el uso de la medicación de rescate ${ }^{16}$.

En una segunda fase, que podríamos llamar de control compartido de la enfermedad, se puede profundizar en el conocimiento de los factores desencadenantes de sus crisis, el niño y la familia puedan optar por diversas alternativas terapéuticas en función de las circunstancias, incluyendo el inicio de corticoide oral si valoran una crisis como moderada.

En esta fase también es importante incidir en el estilo de vida más favorable y la importancia de la práctica deportiva. Este nivel puede ser alcanzado por la mayor parte de las familias y los niños.
En una fase de mayor autocontrol, que no es accesible para todas las familias, el niño o su familia a través de un diario de síntomas o de un registro de pico-flujo, es capaz de controlar su enfermedad de forma autónoma, adaptando la medicación en función del control de la enfermedad; el papel de los sanitarios sería menos relevante, aunque necesariamente presente con visitas de seguimiento que permitan el refuerzo positivo de los logros adquiridos, el control de la función pulmonar y la valoración de la adherencia al tratamiento.

\section{¿Qué metodología usamos para hacer educación?}

Existen excelentes trabajos que nos explican cómo debemos abordar la educación terapéutica ${ }^{15,17}$, y en todos ellos se describe de forma minuciosa el ciclo educativo (figura 1). Para resumirlo de forma práctica, lo primero que debe hacer el educador es conocer qué idea previa tiene de la enfermedad la familia, qué factores cree que influyen en la evolución y los temores que puedan tener acerca de los efectos del tratamiento.

El sanitario pasa de un "rol de experto" que trasmite información a un paciente pasivo, a un modelo mucho más "horizontal" en el que escucha las necesidades y la vivencia de la enfermedad 


\section{Figura 1. Secuencia educativa.}

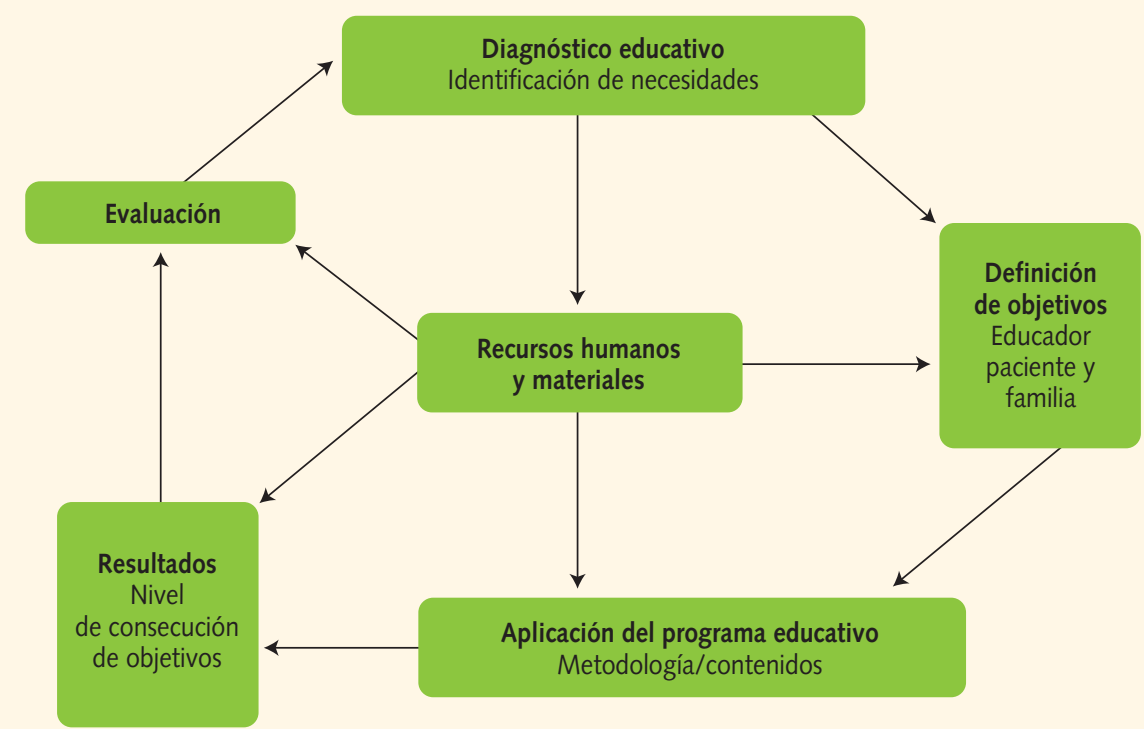

Tomada de: Educación terapéutica en el asma (Korta J, Valverde J, Praena M, Figuerola J, Rodríguez CR, Rueda S, y cols. An Pediatr (Barc). 2007:66(5):500).

del niño y su familia, establece una "alianza terapéutica" pactando cambios de hábitos y modificaciones de conducta y permitiendo una mayor autonomía del paciente, lo que no puede ser percibido como una pérdida de control, sino como un medio de mantener el seguimiento y llegar a una situación en la que los mayores conocimientos del paciente contribuyen a aumentar la eficacia de los tratamientos.

En este proceso educativo pueden establecerse diferencias entre lo que se considera adecuado por parte del sanitario y lo que la familia o el paciente hacen en realidad. Si el sanitario intenta imponer sus ideas, probablemente la relación medico-paciente se resentirá y no se conseguirá "la alianza terapéutica". En vez de imponer se debe intentar concordar ${ }^{17}$ si es preciso, aceptando de forma temporal una actitud o tratamiento que no es del todo correcto, para poder reconducirlo posteriormente.

El plan educativo debe evaluarse de forma periódica, no solamente para comprobar los resultados obtenidos, sino para determinar las nuevas necesidades en la progresión de conocimientos y habilidades de la familia y el niño ${ }^{18}$. 
¿Qué herramientas podemos usar en AP?

Los distintos programas, planes, guías y protocolos implantados en las comunidades aportan diversos materiales más o menos completos para la educación del niño asmático, algunos verdaderamente muy elaborados como los incluidos en el programa de Asturias o el material educativo del Hospital de Donostia, o diseños como la cartilla del niño asmático del programa de Asturias o el documento de salud del asma en Pediatría de Aragón que se realizó remedando la cartilla de salud infantil con información útil para el sanitario y las familias que permite que el niño lleve anotado sus desencadenantes, las crisis que ha sufrido y las pruebas diagnósticas, espirometrías, picos, flujos y tratamientos. Todos ellos pueden consultarse en la página www.respirar.org ${ }^{19}$.

En esta página, además de estas guías, existe la posibilidad de descargar herramientas muy útiles de forma libre para la consulta de la enfermera y el pediatra de AP:

- El modelo tridimensional de los tres tubos que permite una explicación de la enfermedad, la inflamación mantenida y lo que ocurre en el bronquio durante la crisis.
- El póster de inhaladores que descargado en el ordenador de la consulta puede permitirnos, incluso con las familias con mayores dificultades, identificar el tratamiento que sigue el niño y explicarle la acción de los distintos fármacos.

Muchas sociedades científicas pediátricas (neumología, alergología, AEPAP, AEP...) tienen en la red material de acceso libre para educación y diversas guías para padres, que podemos usar en la consulta según nuestras preferencias, o la edad y características de nuestros pacientes.

Existen también numerosos recursos educativos en las asociaciones de pacientes asmáticos (ASBA, ASGA...) y algunos tutoriales interactivos en castellano que podemos recomendar a las familias ${ }^{20}$.

En conclusión, la lista de recursos para la educación en los niños asmáticos de la que disponemos es casi interminable y está demostrado que la educación contribuye de forma importante al control de la enfermedad; únicamente nos queda aplicar la frase de Goethe a nuestro trabajo diario: "Saber no es suficiente; debemos aplicarlo. Estar dispuesto a hacer no es suficiente, debemos hacerlo". 


\section{Bibliografía}

1. Guía clínica de manejo del asma bronquial en niños y adolescentes de Cantabria en Atención Primaria. Santander: Servicio Cántabro de Salud. Consejería de Salud del Gobierno de Cantabria; 2005.

2. Programa de atención al niño asmático de Aragón. Zaragoza: Servicio Aragonés de Salud. Consejería de Salud del Gobierno de Aragón; 2004.

3. Guía para la atención de los niños y adolescentes con asma. Servicio de Salud de Castilla y León. Junta de Castilla y León; 2004.

4. Plan regional de atención al niño y adolescente con asma. Consejería de Salud. Gobierno de Asturias; 2002.

5. Proceso asistencial integrado. Asma en la edad pediátrica. Sevilla: Consejería de Salud. Junta de Andalucía; 2002.

6. Plan de asma infantil de las Islas Baleares. Consejería de Salud y Consumo. Gobierno de las Islas Baleares; 2007.

7. Guía de práctica clínica sobre asma. Servicio Vasco de Salud. Consejería de Sanidad del Gobierno Vasco; 2005.

8. Guía de la práctica clínica de asma infantil de Cataluña. Barcelona: Instituto Catalán de la Salud; 2009 [consultado el 04/09/2009]. Disponible en www.gencat.net/ics

9. GEMA 2009. Guía española para el manejo de asma [consultado el 04/09/2009]. Disponible en www.gema.com

10. Castillo JA, De Benito J, Escribano A, Fernández M, García de la Rubia S, Garde S, y cols. Consenso sobre tratamiento del asma en pediatría. An Pediatr (Barc). 2007;67:253-73.

11. Bacharier LB, Boner $A$, Carlsen $K \mathrm{H}$, Eigenmann PA, Frischer T, Götz $M$, et al. The European Paediatric Asthma Group. Diagnóstico y trata- miento del asma en los niños y adolescentes. Informe del consenso de Practall. Allergy. 2008;63:534.

12. The global strategy for the diagnosis and management of asthma in children 5 years and younger. Global Initiative for asthma (GINA 2009) [consultado el 04/08/2009]. Disponible en www. ginasthma.org

13. Expert Panel Report. EPR-3.2007 Guidelines for the diagnosis and management of asthma [consultado el 08/09/2009]. Disponible en www. nhlbi.nih.gov/guidelines/astma/asthgdh.htm

14. British guideline on the management of asthma. A national clinical guideline. Rev 2009. Brithish Thoracic Society. Routine primary care [consultado el 10/08/2009]. Disponible en www. brit-thoracic.org.uk

15. Praena $M$. Educación en el ámbito de la Atención Primaria. Asma y Educación 2009. Educadores en asma [consultado el 15/08/2009]. Disponible en www.neumped.org

16. Wolf FM, Guevara JP, Grum CM, Clark NM, Cates CJ. Educational interventions for asthma in children (Cochrane Review). In: The Cochrane Library, Issue 1, 2004. Chichester, UK: John Wiley \& Sons, Ltd.

17. Román JM, Korta J, Neira A, Martínez M. Educación y autocuidados en el asma. En: Cobos N, Pérez-Yarza EG. Tratado de neumología infantil. Madrid: Ergon; 2003. p. 635-57.

18. Korta J, Valverde J, Praena M, Figuerola J, Rodríguez $\mathrm{CR}$, Rueda $\mathrm{S}$, y cols. Educación terapéutica en asma. Grupo de trabajo de Asma y Educación de la Sociedad Española de Neumología Pediátrica. An Pediatr (Barc). 2007;66(5):496517.

19. Díaz Vázquez CA. Educación sanitaria en el asma/herramientas educativas [consultado el 10/09/2009]. Disponible en www.respirar.org [actualizado el 25/11/2005]. 
Moneo Hernández I, y cols. Herramientas en la educación del niño asmático

20. Tutoriales Medline Plus. Tutoriales interacti- ble en www.nlm.nih.gov/medlineplus /spanish/tu vos de salud [consultado el 01/09/2009]. Disponi- torial.htlm

\section{ख్య}

